\title{
HÉMOCHROMATOSE ET DIABÈTE
}

\author{
V. Thielen (1), N. Paquot (2), A.J. Scheen (3)
}

RÉSUMÉ : La prise en charge actuelle de l'hémochromatose a considérablement modifié le mode de présentation de la maladie. Aussi, le clinicien est, de plus en plus, amené à évoquer le diagnostic chez des personnes monosymptomatiques. Le diabète est une complication précoce et fréquemment inaugurale de l'hémochromatose. C'est pourquoi, il est important d'en connaître les caractéristiques cliniques et biologiques pour évoquer le diagnostic à son stade le plus précoce. Un dépistage systématique doit, d'ailleurs, être fait devant des présentations atypiques.

\section{INTRODUCTION}

L'hémochromatose héréditaire $(\mathrm{HH})$ est une affection fréquente du métabolisme du fer (avec une prévalence de $0.5 \%$ ) caractérisée par une augmentation de l'absorption intestinale du fer et son dépôt au sein de nombreux parenchymes comme le foie, le pancréas, le cœur, l'hypophyse, mais aussi la peau et les articulations. En l'absence de traitement, la surcharge en fer sera responsable de dommages cellulaires d'évolution progressive avec comme conséquences tardives : cirrhose, hépatocarcinome, diabète, cardiomyopathie, hypogonadisme, arthrose, mélanodermie. Actuellement, le diagnostic présymptomatique a fortement modifié le phénotype des patients hémochromatosiques. La cirrhose est devenue beaucoup plus rare et on est souvent confronté à des patients paucisymptomatiques. Toutefois, le diabète est une complication importante et fréquente de l'hémochromatose. La prévalence du diabète, toutes formes confondues, est de $4 \%$ et son incidence dans l'hémochromatose est approximativement de $55 \%$ chez les patients ayant des signes cliniques de la maladie et seulement de $17 \%$ chez ceux qui ne présentent que des stigmates biologiques. Ce chiffre est de $80 \%$ chez les patients cirrhotiques. Un diagnostic plus précoce et une prise en charge plus adéquate de l'hémochromatose a permis de diminuer la proportion de sujets déjà diabétiques lors du diagnostic de 55 à $30 \%$ entre 1975 et 1990 (1). Cependant, le diabète compliquant 1'hémochromatose doit, dès à présent, faire partie du diagnostic différentiel d'un diabète inaugural en l'absence d'étiologie évidente. Son diagnostic précoce est important étant donné que l'instauration d'un traitement adéquat par saignées permet d'arrêter l'évolution de la maladie et même parfois de l'améliorer $(2,3)$.

(1) Etudiante $4^{\text {ème }}$ doctorat

(2) Agrégé, Chef de Clinique associé, (3) Professeur, Chef de Service, Service de Diabétologie, Nutrition et Maladies métaboliques, Département de Médecine, CHU Sart Tilman, Liège

\section{HEMATOCHROMATOSIS AND DIABETES}

SUMMARY : Nowadays, haemochromatosis is often diagnosed when the patient is monosymptomatic. Diabetes is frequently the first expression of the disease. So, it is important to know the clinical and biologic characteristics to evoke diagnosis as early as possible. Uncommon presentations request systematic screening.

KEYWORDS : Secondary diabetes - Insulinresistance

\section{RAPPEL：LA DÉMARCHE DIAGNOSTIQUE}

Le diagnostic de l'hémochromatose n'est pas un diagnostic facile. Les premiers signes cliniques sont aspécifiques (asthénie, malaise,...). En cours d'évolution de la maladie, la mise au point d'un diabète ou d'une hépatopathie peut amener au diagnostic. Aussi, le diagnostic précoce est souvent une découverte biologique (fer et ferritine élevés). Une saturation de la transferrine supérieure à $50 \%$ reste actuellement le meilleur test de dépistage ou de confirmation d'une suspicion clinique ou biologique (4). Le diagnostic d'HH peut alors être renforcé par la réalisation d'examens complémentaires. L'IRM est l'examen d'imagerie de référence pour l'HH (4). L'analyse anatomopathologique d'une biopsie hépatique avec quantification du fer intrahépatique demeure dans bien des cas essentielle (2). Par ailleurs, la recherche des trois mutations principales de 1'HH (C282Y, H63D, S65C) permet d'affiner au maximum la suspicion clinique (5). En présence d'un test génétique négatif avec un phénotype typique, il faut considérer la possibilité (environ $10 \%$ ) d'être en présence d'un faux négatif, étant donné que tous les allèles impliqués ne sont pas recherchés (tableau I).

TABlEAU I. StratÉGIE POUR ÉTABLIR LE DIAGNOSTIC D'HÉMOCHROMATOSE.

Suspicion diagnostique

Signes cliniques, biologie de routine (fer, ferritine)

Affirmer la surcharge en fer au niveau biologique Saturation de la transferrine $>50 \%$

Confirmer la surcharge en fer via des examens complémentaires IRM, biopsie hépatique

Affirmer la nature génétique de l'hémochromatose anamnèse familiale, recherche des trois mutations principales $(\mathrm{C} 282 \mathrm{Y}$, H63D, S65C)

\section{Pathogénèse}

Les principaux mécanismes pathogéniques conduisant à l'intolérance glucidique et au diabète dans l'HH sont à ce jour mieux connus. De nombreux arguments épidémiologiques, immunologiques et histologiques prouvent, en tout cas, que le diabète de l'HH est indépendant du diabète de type 1 et de type 2 . Par contre, il est admis que le fer est diabètogène. C'est ce que 
démontre plusieurs études expérimentales basées sur des modèles animaux avec administration orale ou intrapéritonéale de fer, mais aussi quelques travaux intéressants qui décrivent le développement progressif d'un diabète chez les malades atteints d'hémochromatose secondaire post-transfusionnelle et son amélioration après chélation par la desferroxamine (4). L'excès de fer a une toxicité propre et contribue au développement de l'hyperglycémie par deux mécanismes principaux. D'abord, les dépôts de fer dans les cellules B des îlots de Langerhans provoquent une réduction de la sécrétion d'insuline. Il en résulte donc un état d'insulinopénie. L'autre conséquence d'un excès de fer est une insulinorésistance liée au dépôt de fer intrahépatique qui aboutit à une altération de l'action de l'insuline à ce niveau. Ainsi, chacun de ces deux mécanismes isolément et/ou leur combinaison conduit progressivement de la normoglycémie avec hyperinsulinémie (insulinorésistance avec hypersécrétion compensatoire) à l'intolérance au glucose, puis au diabète non insulinoréquérant et, enfin, insulinoréquérant (4).

\section{Caractéristiques CliniQues et BIOLOGIQUES DU DIABÈTE}

Les principales complications de l'hémochromatose sont le diabète et la cirrhose hépatique et apparaissent généralement vers 1'âge de 50 ans. Grâce à l'amélioration du dépistage présymptomatique, la cirrhose est devenue rare au moment $\mathrm{du}$ diagnostic et c'est souvent par l'observation d'un diabète inaugural, sans facteurs génétiques ou environnementaux prédisposants, que l'hémochromatose est découverte. Le diabète dans l'HH est caractérisé par un début plus précoce que dans le diabète de type 2 (45-50 ans). Il n'est pas fréquemment associé à l'obésité, contrairement au diabète de type 2 au cours duquel on observe un surpoids dans $80 \%$ des cas. On note un sexe ratio $\mathrm{H} / \mathrm{F}$ de 10/1 (6). Par ailleurs, on ne retrouve pas la présence de marqueurs d'autoimmunité vis-à-vis des îlots de Langerhans. La prévalence des deux maladies est estimée à $0,5 \%$ pour l'hémochromatose et à $4 \%$ pour le diabète.

Toutes les complications du diabète, micro(rétinopathie, néphropathie) et macrovasculaires (répercussions cardiovasculaires), ont été décrites dans le diabète secondaire à l'hémochromatose. Toutes sont aussi fréquentes chez les patients diabétiques de type 1 que chez les hémochromatosiques apparentés pour l'âge et la durée d'évolution du diabète, à l'exception de l'hypertension artérielle qui semble moins fréquente (7). De plus, certaines apparaissent moins sévères, comme la rétinopathie qui est rarement décrite comme étant proliférative. Par ailleurs, Paris et coll. ont mis en évidence, lors d'une étude clinique la présence de microangiopathie et de neuropathie chez $50 \%$ des sujets hémochromatosiques au moment du diagnostic (4).

Sur le plan biologique, le premier signe de l'anomalie du métabolisme glucidique est 1'intolérance au glucose. Lors d'un diagnostic plus tardif dans l'évolution de la maladie, la destruction progressive des cellules B, exposées au stress oxydatif de la surcharge en fer, conduit à une déficience d'insulinosécrétion. Celle-ci peut être mise en évidence par la réduction de la sécrétion de Cpeptide en réponse à l'injection de glucagon. On a également observé que les patients diabétiques présentant simultanément une surcharge en fer hépatique ont une insulinorésistance précoce. La prise orale de glucose ou encore l'injection de glucagon est associée à une élévation exagérée d'insuline plasmatique alors que la glycémie reste normale. Cependant, cette hyperinsulinémie n'est pas associée à une hypersécrétion d'insuline, étant donné que les concentrations du C-peptide restent normales. L'accumulation de fer dans les hépatocytes explique probablement la diminution de la clairance hépatique de l'insuline. En conséquence, l'incidence du diabète est plus élevée en présence d'une surcharge en fer hépatique. Il faut noter, cependant, que les observations actuelles ont démontré qu'un diabète sucré peut survenir indépendamment d'une cirrhose, de même qu'un patient cirrhotique peut rester indemne de diabète. L'ensemble de ces constatations suggère que les facteurs modulant la génèse et l'expression d'un diabète dans 1'HH sont complexes et variables. Quoi qu'il en soit, son développement dans une $\mathrm{HH}$ ne semble pas lié au génotype, la fréquence de l'homozygotie C282Y étant identique chez des sujets diabétiques et non diabétiques (4).

\section{LES PARTICULARITÉS DU TRAITEMENT}

Le traitement du diabète secondaire ne diffère pas de celui des autres formes de diabète : prise en charge diététique, antidiabétiques oraux, insuline. Cependant, le taux d'HbA1c à l'admission est, en général, plus élevé $(8,5 \%)$ que les valeurs observées dans le registre "convention INAMI diabète de type 1" (4). Le développement potentiel des complications doit encourager une optimalisation afin de prévenir ou de retarder leur développement. Le diabète insulinorequérant, qui représente l'évolution quasi systématique de ces patients, impose souvent le recours à des doses élevées d'insuline étant donné la résistance à l'insuline fréquemment associée. 
La prise en charge appropriée de ces patients nécessite l'évaluation et le traitement de la surcharge en fer. Une correction de celle-ci permet, en effet, non seulement de retarder au maximum la survenue des autres complications de la surcharge en fer, mais aussi d'arrêter l'évolution, voire d'améliorer, les maladies de surcharge préexistantes. L'hémochromatose est traitée par la réalisation de phlébotomies de 300-500 $\mathrm{ml}$ par semaine jusqu'à ce que les signes biologiques de déplétion martiale soient mis en évidence, tels que la diminution du taux de ferritine sérique mais aussi la normalisation du fer sérique. La chute du taux plasmatique d'hémoglobine est également un facteur déterminant. Ensuite, on réalise un traitement par phlébotomie tous les 23 mois, visant à maintenir la ferritine sérique à une valeur seuil $(50 \mu \mathrm{g} / 1)$. Rarement, lorsqu'on souhaite une amélioration drastique de la surcharge en fer (par exemple, s'il existe une cardiopathie sévère avec décompensation cardiaque), on réalise en complément de la phlébotomie des injection de desferroxamine. Les effets de la déplétion en fer sont variables. En ce qui concerne le métabolisme du glucose, le diabète non insulinodépendant est généralement stabilisé et même amélioré. Par contre, l'évolution du diabète insulinorequérant n'est pas modifiée par les phlébotomies. D'ailleurs, comme le montrent deux études cliniques, les doses journalières d'insuline restent inchangées $(4,7)$.

La destruction des cellules B est un processus dynamique et réversible jusqu'à un certain niveau de surcharge en fer. Si la correction survient avant la destruction complète et irrréversible des cellules $\mathrm{B}$, une amélioration clinique sera mise en évidence.

\section{DÉPISTAGE SYSTÉMATIQUE ET DIABÈTE}

Les avis sont divergents à ce propos. Certains ne recommandent pas la recherche systématique d'une hémochromatose chez tout patient présentant un diabète isolé. L'argument avancé est la mise en évidence d'une prévalence identique de l'hémochromatose chez les sujets diabétiques et non diabétiques (1). A l'inverse, ils préconisent cette recherche chez les patients présentant un diabète et une hépatopathie, étant donné que le risque d'être porteur d'une hémochromatose est alors 20 à 30 fois supérieur à celui des patients présentant uniquement un diabète. Ce dépistage sera systématique et ce, même s'il existe un facteur aggravant la maladie, comme une intoxication éthylique associée (1). La recherche d'une hémochromatose chez les sujets présentant un diabète d'expression tardive sans surpoids paraît justifiée. La surcharge en fer pourrait fournir le facteur d'insulinorésistance nécessaire au développement clinique du diabète (1). D'autres préconisent d'envisager, dans le diagnostic différentiel d'une hyperglycémie récente, la possibilité d'un diabète secondaire à l'hémochromatose, à côté des diabètes de type 1 et de type 2 (4). Ils affirment, quant à eux, la fréquence élevée de diabète dans l'HH. La démarche clinique quotidienne doit tenir compte de ces deux points de vue. Sans tomber dans l'excès d'un diagnostic systématique de l'hémochromatose lors de la mise au point d'un diabète de type 1 ou 2 classique, il faut, dès à présent, l'évoquer devant une présentation atypique (âge moyen de 40-50 ans, absence de surpoids dans le type 2 , absence d'autoanticorps dans le type 1) Cette attitude permet une prise en charge précoce et la mise en place d'une prévention secondaire des lésions systémiques.

\section{Conclusion}

Le diabète reste une complication fréquente de l'hémochromatose. Il doit donc faire partie du diagnostic différentiel d'un diabète inaugural en présence d'un phénotype atypique (patient d'âge moyen et de poids normal). Son dépistage précoce est important étant donné que l'instauration d'un traitement adéquat par saignées permet d'améliorer, non seulement l'évolution de la maladie, mais aussi l'équilibre glucidique.

\section{BIBLIOGRAPHIE}

1. Dubois-Laforgue D, Larger E, Timsit J.- Faut-il rechercher une hémochromatose lors de la découverte d'un diabète ? Diabetes Metab, 2000, 26, 318-321

2. Kowdley KV, Tait JF, Bennett RL, et al.- Hereditary Hemochromatosis. Geneclinics, 2000, 151, 67-75.

3. Hanson EH, Imperatore G, Burke W.- HFE gene and hereditary hemochromatosis : review article. Am J Epidemiol, 2001, 154, 193-206.

4. Paris I, Hermans M, Buysschaert M.- Les complications endocriniennes de l'hémochromatose génétique. Acta Clin Belgica, 1999, 54, 334-345.

5. Barton JC, Sawada-Hirai R, Rothenberg BE, et al.Two novel missense mutations of the HFE gene and identification of the S65C mutation in Alabama hemochromatosis probands. Blood Cells Mol Dis, 1999, 25, 147-155.

6. Buysschaert M, Paris I, Selvais PH.-Clinical aspects of diabetes secondary to idiopathic haemochromatosis in French-speaking Belgium. Diabetes Metab, 1997, 23, 308-331.

7. Yaouanq JM.- Diabetes and haemochromatosis : current concepts, management and prevention. Diabetes Metab, 1995, 21, 319-329.

Les demandes de tirés à part sont à adresser au Dr N. Paquot, Service de Diabétologie, Nutrition et Maladies métaboliques, Département de Médecine, CHU Sart Tilman, 4000 Liège. 